\title{
POLYMORPHISMS DETECTED IN THE TYROSINASE AND MATP (SLC45A2) GENES DID NOT EXPLAIN COAT COLOUR DILUTION IN A SAMPLE OF ALPACA (VICUGNA PACOS) \\ R. Cransberg and K. A. Munyard
}

\author{
Alpaca Molecular Research Group, School of Biomedical Science, Curtin University of \\ Technology, Perth, WA 6102
}

\section{SUMMARY}

The molecular basis of inheritance of alpaca fibre colour is poorly understood. However, colour dilution genes are anticipated to be causing a major effect on alpaca fibre colour. Candidate genes for dilutions included tyrosinase (tyr) and membrane associated transport protein (matp), both of which have been associated with coat colour dilution in other species. The coding regions of the tyr and matp genes were sequenced in 24 animals with various colour phenotypes. No polymorphism found in the coding region of tyr and matp exons 1, 3, 4, 5 and 7 could account for the dilutions in fibre colour observed.

\section{INTRODUCTION}

Alpaca fibre is highly valued because it is strong and suitable for use in high quality clothes, fabrics and carpets (Frank et al. 2006). White alpaca fibre is considered the most valuable because it is easy to dye, thus coloured fibres only sell for about $65 \%$ of the price of white fibres (Frank et al. 2006). However there are not enough white alpacas in Australia to meet market demand, with only approximately a third of alpacas being light enough to be classed as white (Paul 2006). This project examined the tyr and matp genes in alpacas, located at the albino and underwhite loci respectively. The aim was to identify polymorphisms that occur within these genes and ascertain if any of these can be consistently linked to a particular colour phenotype. We hypothesise that polymorphisms in these genes may result in a structural change, or a change in expression levels which will therefore alter the quantity of melanin produced, and result in a coat colour phenotype which is lighter or darker than that which would be expected of the wildtype individual.

\section{MATERIALS AND METHODS}

Samples. Blood (approximately $5 \mathrm{~mL}$ ) was taken from 24 alpacas of both sexes, with a variety of different colour phenotypes, from various herds across Western Australia, New South Wales and Victoria. The colour variants selected placed an emphasis on potentially diluted phenotypes such as pink skin (PS) white and PS light fawn and also included non-fading and fading black, black and tan, bay, chestnut, black skin (BS) brown, PS rosegrey, BS silvergrey, BS white and BS blueeyed white.

DNA Extraction. DNA was extracted from alpaca blood using the salt precipitation method described by Miller et al. (1988) Where this method failed to yield sufficient quantities of DNA, or where the quantity of blood obtained was insufficient for the salt precipitation method, the DNeasy blood and tissue DNA extraction kit (Quigen) was used, according to the manufacturer's instructions.

Primer Design and Amplification. Alpaca tyr and matp sequences were retrieved from trace archive files deposited in GenBank (NCBI) where possible, using BLAST analysis against known cattle exon sequences. Primers were then designed in regions flanking each exon. Where the trace archive sequence was incomplete (for matp exons 1,2 and 6) a comparative genetics approach was 
adopted. Sequences from the cattle, dog, human and mouse genomes were aligned and primers were designed using sequence from consensus regions flanking each exon.

Genomic DNA (50-100ng) was used as template for PCR using $2 \mu \mathrm{M}$ of forward primer, $2 \mu \mathrm{M}$ reverse primer, $1 \times$ Polymerisation buffer (Fisher biotec), $2 \mathrm{mM} \mathrm{MgCl}_{2}$ (Fisher biotec) and $0.75 \mathrm{u}$ BIOTAQ polymerase (Bioline) in a $10 \mu \mathrm{L}$ reaction. Optimized amplification conditions were: $95^{\circ} \mathrm{C}$ for $2 \mathrm{~min} ; 30-40$ cycles of $95^{\circ} \mathrm{C}$ for $20 \mathrm{~s}, 57-58^{\circ} \mathrm{C}$ for $30 \mathrm{~s}$ and $72^{\circ} \mathrm{C}$ for $1 \mathrm{~min}$; then $72^{\circ} \mathrm{C}$ for $5 \mathrm{~min}$. tyr exon 1 and matp exons 3,4 and 5 were amplified using 30 cycles with $58^{\circ} \mathrm{C}$ annealing temperature; tyr exons 2,4 and 5 were amplified using 30 cycles with $57^{\circ} \mathrm{C}$ annealing temperature; tyr exon 3 was amplified using 40 cycles at $58^{\circ} \mathrm{C}$ annealing temperature, matp exon 1 was amplified using 35 cycles at $57^{\circ} \mathrm{C}$ annealing temperature; and matp exon 7 was amplified using 35 cycles at $58^{\circ} \mathrm{C}$ annealing temperature.

DNA Sequencing. PCR products were amplified from genomic DNA in five separate $10 \mu \mathrm{L}$ reactions and pooled prior to sequencing. Sequencing was performed using the ABI Big Dye Terminator ${ }^{\circledR}$ system at either Murdoch University, Perth using a 48-capillary ABI 3730 DNA analyser or at Macrogen, Korea, using 96-capillary ABI 3730XL DNA analysers.

Analysis. Vector NTI software (Invitrogen) was used to visualise and analyse sequencing results and SpliceView (ORIEL) was used to aid in determining intron/exon boundaries for predicted proteins. Statistical analyses were performed using the JMP ${ }^{\circledR} 7$ statistical package (SAS institute Inc. 2007). The Pearson $\chi^{2}$ was used to investigate if an association existed between fibre colour and SNP's or nucleotides. Statistical significance was accepted at $\mathrm{p} \leq 0.05$.

\section{RESULTS}

The five exons of the tyr gene and five of the seven matp exons were sequenced all 24 animals. While tyr exon 2 was largely sequenced only in one direction due to difficulties sequencing through an adjacent microsatellite, most exons were sequenced in both directions for the majority of alpacas. Ten single nucleotide polymorphisms (SNPs) were detected in the coding region of tyr. Seven of these SNPs were synonymous polymorphisms and three were non-synonymous (Table 1). Two SNPs were detected in the five matp exons that were sequenced. One of these was synonymous and the other was non-synonymous (Table 2). The C1526T mutation was found in seven heterozygotes and 17 homozygote $C$ animals. There was no association between SNP and fibre colour $\left(\chi^{2}=0.14, p=1.0\right)$ for any of the SNPs found in tyr or matp.

Table 1. Single Nucleotide Polymorphisms found in the coding region of tyr

\begin{tabular}{rccc}
\hline Exon & Nucleotide Position & Nucleotide Change & Amino Acid Change \\
\hline Exon 1 & 78 & $\mathrm{C} / \mathrm{A}$ & $\mathrm{NA}$ \\
Exon 1 & 126 & $\mathrm{C} / \mathrm{T}$ & $\mathrm{NA}$ \\
Exon 1 & 162 & $\mathrm{C} / \mathrm{T}$ & $\mathrm{NA}$ \\
Exon 1 & 480 & $\mathrm{C} / \mathrm{T}$ & $\mathrm{NA}$ \\
Exon 1 & 513 & $\mathrm{C} / \mathrm{T}$ & $\mathrm{NA}$ \\
Exon 1 & 784 & $\mathrm{C} / \mathrm{T}$ & $\mathrm{NA}$ \\
Exon 2 & 851 & $\mathrm{G} / \mathrm{T}$ & $\mathrm{Ala} / \mathrm{Ser}$ \\
Exon 5 & 1372 & $\mathrm{C} / \mathrm{T}$ & $\mathrm{NA}$ \\
Exon 5 & 1490 & $\mathrm{G} / \mathrm{T}$ & $\mathrm{Arg} / \mathrm{Leu}$ \\
\hline
\end{tabular}




\begin{tabular}{llll}
\hline Exon 5 & 1498 & T/C & Cys/Arg \\
\hline
\end{tabular}

An alpaca TYR protein sequence was predicted from the five exon sequences, using SpliceView (ORIEL) coupled with comparative genetics using the known cattle coding sequence. This protein was 530 amino acids long; $89.4 \%$ identical to the cattle and $88.1 \%$ identical to the human protein.

Table 2. Single Nucleotide Polymorphisms found in the coding region of matp

\begin{tabular}{rccc}
\hline Exon & Nucleotide Position $^{*}$ & Nucleotide Change & Amino Acid Change \\
\hline Exon 3 & 843 & G/A & NA \\
Exon 7 & 1526 & C/T & Thr/Met \\
\hline
\end{tabular}

* Nucleotide positions are speculative, using cattle sequence to fill the regions of exon 2 and 6

Matp exons 2 and 6 gave multiple amplicons in PCR after extensive optimisation of reaction conditions. The multiple amplicons produced by PCR, from two different animals, were cloned, and three of these clones were sequenced for each animal. However, BLAST searches confirmed that none of these clones contained sequence homologous to matp from any other species.

\section{DISCUSSION}

Tyrosinase. None of the three non-synonymous SNPs found in the tyr gene had any significant correlation with fibre and/or skin colour. The G851T polymorphism occurs around the middle of the protein, and is 53 amino acids away from the nearest glycosylation site or copper binding domain (Schweikardt et al. 2007, Wang and Hebert 2006). The likelihood of this change having little effect is further supported by the observation that serine is present in the same position of the protein in the human and rabbit, while the alanine, more common in alpacas (Table 2), is also present in cattle, dog and chicken (Schmutz et al. 2004).

The G1490T polymorphism causes a change in the last amino acid of the TYR transmembrane region, if the alpaca protein is similar to most known Tyr proteins (Schmutz et al. 2004). However, the alpaca tyr gene encodes for arginine in this position, differing from the genes of the dog, human, mouse and cattle, which all encode serine; the rabbit which encodes glycine; and the chicken which encodes isoleucine (Schmutz et al. 2004). This represents a large biological difference in amino acid, however, no SNP in the cytoplasmic tail of Tyr have been documented to have any effect on coat colour in the extensively studied human and mouse Tyr genes and there was no apparent correlation with a change in alpaca fibre colour either.

The T1498C SNP occurred only three codons after the one previously described. The fact that two non-synonymous SNPs were found close together in this region, suggests that the region is under little selective conservation. The cysteine residue present at this position in the wild-type is highly conserved in Tyr proteins of other species (Schmutz et al. 2004). However the position of this residue in the cytoplasmic tail separates it from other cysteine residues by the melanosomal membrane, therefore this residue does not form any disulfide bonds in the final protein structure of other species (Wang and Hebert 2006).

Membrane Associated Transport Protein. For the non-synonymous C1526T polymorphism identified in the matp gene no homozygote $\mathrm{T}$ animals were observed. This suggests that 
homozygote $\mathrm{T}$ at this position may be non-viable. As seven of the animals were heterozygous for this SNP and the other 17 were homozygote C, the sample group is also clearly not in HardyWeinberg proportions. Given the broad range of animals in the sample group this would appear to be significant, however there is no apparent correlation from this SNP to any given colour phenotype. Based on the Newton et al. (2001) prediction of the human MATP protein, this polymorphism will lie in the $12^{\text {th }}$ and final transmembranous region. This region is highly conserved among human, mouse, cattle and alpaca coding regions. Given that the polymorphism lies within a transmembranous region, the change from threonine to a non-polar, hydrophobic methionine may prove to have little effect. Indeed a similar threonine for proline substitution was reported by Inagaki et al. (2004) earlier in the same transmembranous region in humans, which was identified as a population variant, with no significant effect on hair colour.

\section{CONCLUSION}

A sample group of 24 alpacas yielded a total of ten SNPs in the coding region of the tyr gene, while two SNPs were found in five of the seven exons of matp. None of these SNPs correlated with a detectable change in colour within the sample group. However, our spectrophotometric studies have strongly suggested that colour dilution genes play a major role in alpaca pigmentation (data not shown). Key regulatory regions of the tyr and matp genes will have to be examined thoroughly before these genes are confirmed as not playing a significant role in alpaca pigmentation.

\section{ACKNOWLEDGMENTS}

This work was funded largely by the Rural Industry Research and Development Corporation (RIRDC).

\section{REFERENCES}

Frank, E N, Hick, M V H, Gauna, C D, Lamas, H E, Renieri, C \& Antonini, M (2006) Phenotypic and genetic description of fibre traits in South American domestic camelids (llamas and alpacas), Small Ruminant Research, 61: 113-129.

Inagaki, K, Suzuki, T, Shimizu, H, Ishii, N, Umezawa, Y, Tada, J, Kikuchi, N, Takata, M, Takamori, K, Kishibe, M, Tanaka, M, Miyamura, Y, Ito, S \& Tomita, Y (2004) Oculocutaneous Albinism Type 4 Is One of the Most Common Types of Albinism in Japan, The American Journal of Human Genetics, 74: 466-471.

Miller, S A, Dykes, D D \& Polesky, H F (1988) A simple salting out procedure for extracting DNA from human nucleated cells, Nucleic Acids Research, 16: 1215.

Newton, J M, Cohen-Barak, O, Hagiwara, N, Gardner, J M, Davisson, M T, King, R A \& Brilliant, M H (2001) Mutations in the Human Orthologue of the Mouse underwhite Gene (uw) Underlie a New Form of Oculocutaneous Albinism, OCA4, The American Journal of Human Genetics, 69: 981-988.

Paul, E (2006) Colour Review. Australian Alpaca Association National Conference. Adelaide.

Schmutz, S M, Berryere, T G, Ciobanu, D C, Mileham, a J, Schmidtz, B H \& Fredholm, M (2004) A form of albinism in cattle is caused by a tyrosinase frameshift mutation., Mammalian Genome, 15: 62-67.

Schweikardt, T, Olivares, C, Solano, F, Jaenicke, E, Garcia-Borron, J C \& Decker, H (2007) A three-dimensional model of mammalian tyrosinase active site accounting for loss of function mutations, Pigment Cell Research, 20: 394-401.

Wang, N \& Hebert, D N (2006) Tyrosinase maturation through the mammalian secretory pathway: bringing color to life, Pigment Cell Research, 19: 3-18. 